\title{
AUTOMATED OPTICAL FIBER ALIGNMENT IN 2-AXES USING 3D SHAPED ACTUATORS
}

\author{
Brian Morgan and Reza Ghodssi \\ MEMS Sensors and Actuators Lab (MSAL) \\ Department of Electrical and Computer Engineering, Institute for Systems Research \\ University of Maryland, College Park, MD USA \\ Email: ghodssi@eng.umd.edu
}

\begin{abstract}
We report the development and characterization of an automated on-chip 2-axis optical fiber alignment system using 3D shaped actuators. Gray-scale technology was used to integrate sloped silicon wedges with electrostatic comb-drive actuators to deflect an optical fiber cantilever in- and out- of the plane of the wafer. Automated fiber alignment to Indium-phosphide (InP) waveguides with an accuracy of $1.6 \mu \mathrm{m}$ or better is demonstrated for the first time. The influences of alignment target location, actuation parameters, and alignment algorithms on total alignment time are also presented.
\end{abstract}

\section{INTRODUCTION}

Alignment of an optical fiber within an optoelectronic package is an on-going challenge and often dominates optoelectronic module cost. Passive fiber alignment techniques would be preferred for their simplicity where passive systems utilizing silicon waferboards have reported alignment accuracies of $1-2 \mu \mathrm{m}$ [1]. Yet, as alignment requirements approach $0.2 \mu \mathrm{m}$ for many applications [2], active alignment is necessary to achieve sufficient coupling. Thus, to alleviate the expensive, slow macro actuators currently required to achieve sub-micron alignment, multi-axis on-chip methods for final alignment of the optical fiber are attractive.

Previous MEMS fiber actuators have required complicated fabrication (LIGA [3]) and/or specialized fiber preparation (e.g. permanent magnets [4]). In contrast, the actuator discussed here requires neither, instead exploiting the coupled motion of opposing in-plane actuators with integrated $3 \mathrm{D}$ wedges. This device creates a dynamic v-groove controlled via simple MEMS in-plane actuators to modify the horizontal and vertical position of the optical fiber, as shown in Figure 1 [5].

This paper characterizes the performance of fabricated optical fiber actuators and demonstrates their potential for automated inpackage alignment. Results regarding the alignment range, resolution, and speed of current devices are reported. The influences of alignment target location, actuation parameters, and alignment algorithm on total alignment time are also evaluated.

\section{DESIGN AND FABRICATION}

The optical fiber aligner discussed here is composed of three primary components, as shown in the top view schematic of Figure 2: a static trench, two sets of opposing sloped alignment wedges, and an in-plane actuation mechanism. An optical fiber is placed in the static trench and secured using UV-curing epoxy. This static trench provides approximate passive alignment of the fiber cantilever so that its free end rests between the sloped alignment wedges. The actuation mechanism can be made using any number of in-plane MEMS actuation techniques, but in this case electrostatic comb-drives were chosen for simplicity. (a)

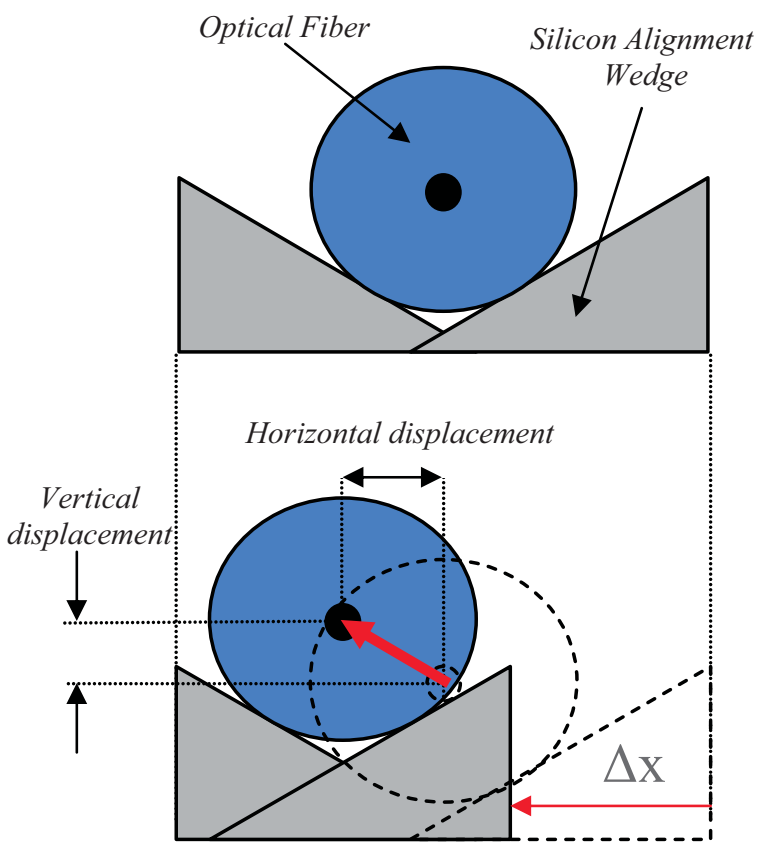

Figure 1: Optical fiber (a) at rest and (b) after actuating single wedge, causing horizontal and vertical displacement [5].

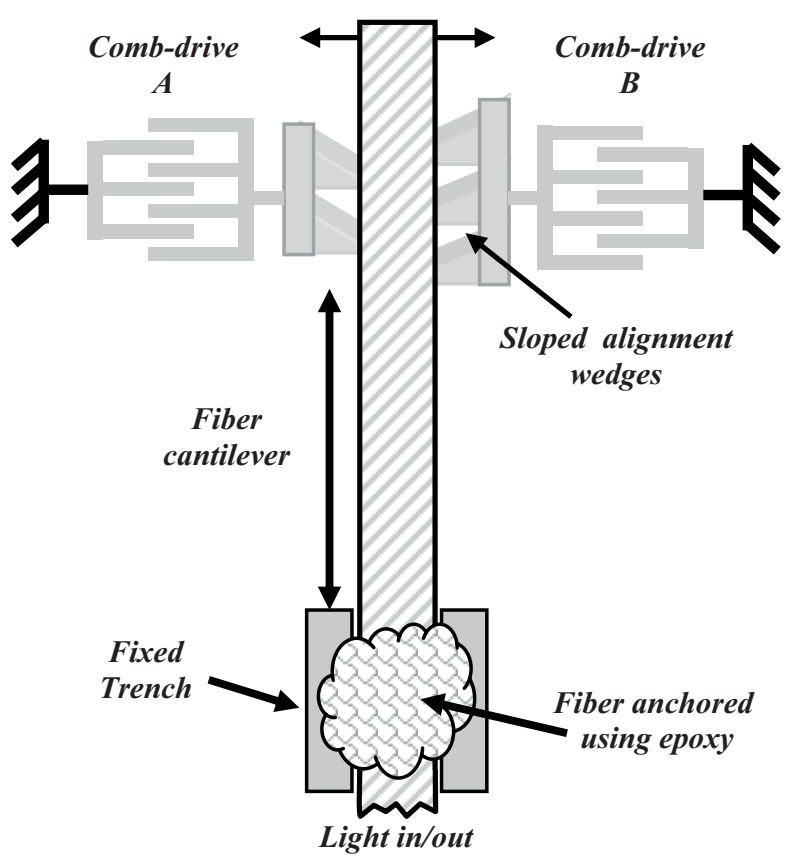

Figure 2: Top view schematic of the 2-axis optical fiber actuator. 


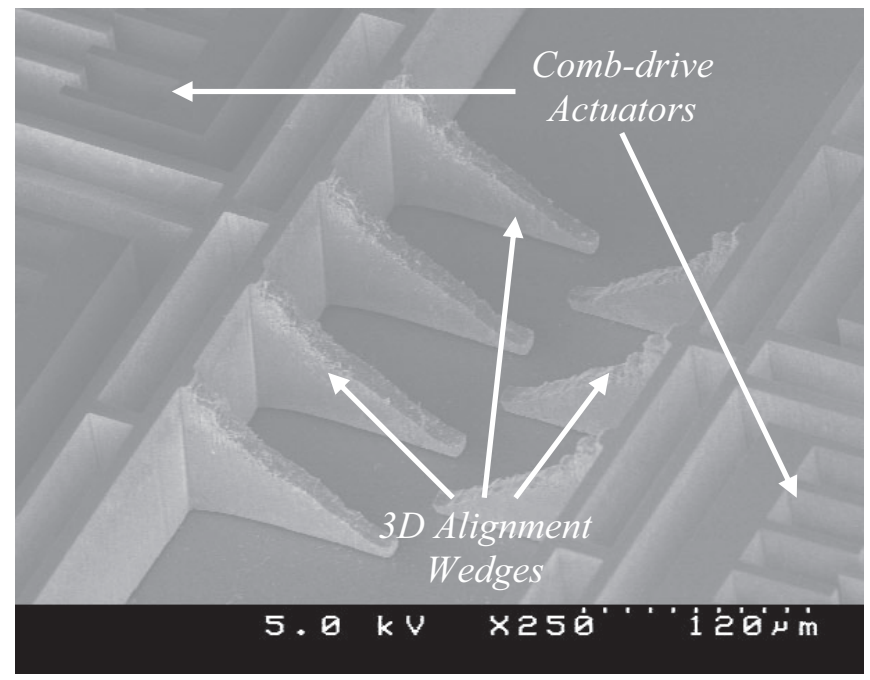

Figure 3: 3D alignment wedges before fiber attachment.

The 3D silicon wedges were fabricated using gray-scale technology, where a single variable intensity exposure creates a $3 \mathrm{D}$ photoresist mask that is transferred into the underlying substrate using deep reactive ion etching (DRIE) [6]. The gray-scale process was integrated with an electrostatic actuator process using SOI wafers, thus creating the suspended 3D wedges that are shown in Figure 3 [5]. Of particular importance is the design and fabrication of the wedges themselves, which must be smooth compared to the size of the optical fiber (diameter $=125 \mu \mathrm{m}$ ). A set of 50 gray-levels was created in photoresist to define the slope. Specifically developed DRIE recipes were then used to control the etch selectivity of silicon to photoresist to define the final vertical dimensions of the 3D structure in silicon. SEM's such as Figure 3 show that micron-level roughness has been achieved over the majority of the slope. Quantitative roughness measurements are difficult on such sloped, high aspect ratio structures.

For final assembly, a single mode optical fiber (SMF-28) was manually stripped and cleaved. The cleaved free end of the fiber was placed between the two wedges that are attached to the combdrives. The bulk fiber was then secured in the static trench with UV-curing epoxy (Norland Products, Inc.) to create a flexible cantilever. A device after fiber attachment is shown in Figure 4. Since this fiber attachment process is entirely manual, it is difficult to ensure that the fiber touches both wedges in its resting state. Reliable operation can be achieved with small gaps between the wedge and fiber, but result in a voltage offset before the fiber actually moves which can slightly complicate fiber control.

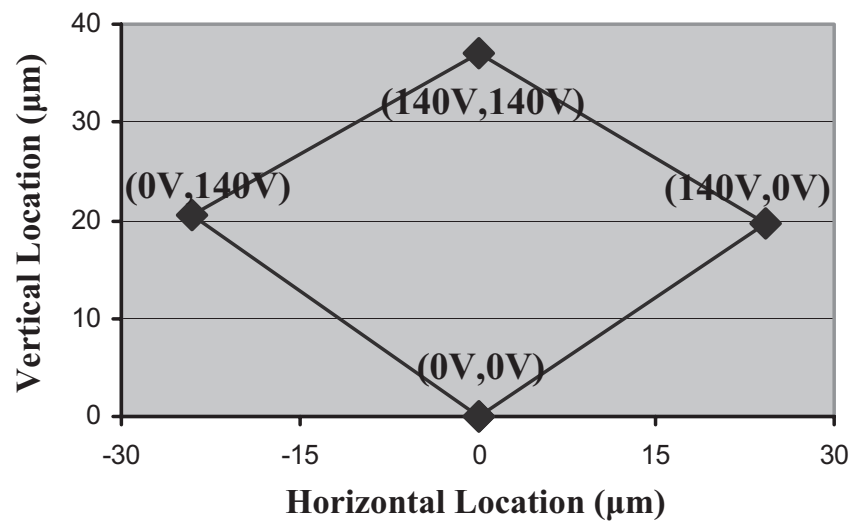

Figure 5: Measured fiber location for extreme actuation voltages, which form a diamond-shaped alignment area.

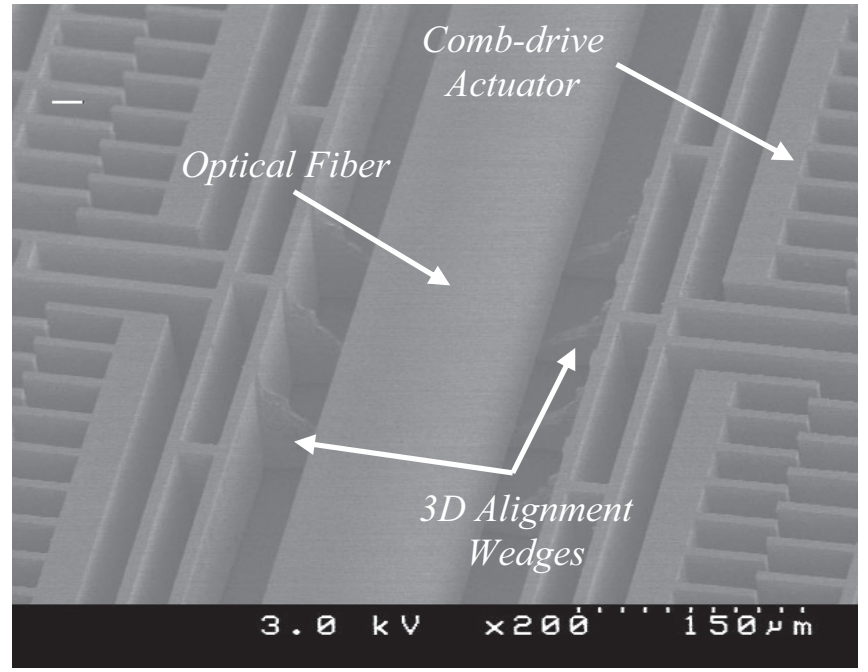

Figure 4: SEM of the device after fiber attachment.

\section{Static Testing}

The extents of fiber movement were investigated by applying four voltage combinations to the two actuators. The fiber tip location was measured using the peak coupling to an opposing electrostrictively controlled fiber. These four points are shown in Figure 5, representing the extreme movements of each alignment wedge. Fiber positions within the diamond-shaped bounds of these measurements ( $37 \mu \mathrm{m}$ tall, $48 \mu \mathrm{m}$ wide) should be achievable given the appropriate set of applied voltages. If one wedge is kept stationary and the other moved, the fiber tip will trace out an angled trajectory parallel to one side of the diamond-shaped alignment area shown in Figure 5.

Since this actuator relies on multiple surfaces sliding together, an important characteristic to investigate is hysteresis of the fiber motion, primarily caused by the morphology of the gray-scale wedges. Thus, a sequence of increasing then decreasing identical voltages was applied to each side of the actuator to create purely vertical fiber deflection. The coupled power to a fixed output fiber (which should be Gaussian in nature for cleaved fiber-fiber coupling) was then measured as the MEMS-actuated fiber moved 'Up' and 'Down' over multiple cycles. As shown in Figure 6, there is a definite hysteresis between the 'Up' and 'Down' actuation paths, amounting to a 'lag' of approximately $4 \mu \mathrm{m}$ between the peaks. We believe that frictional forces between the wedges and optical fiber, increases the force required from each actuator to move the fiber "Up." On the way back "Down," the

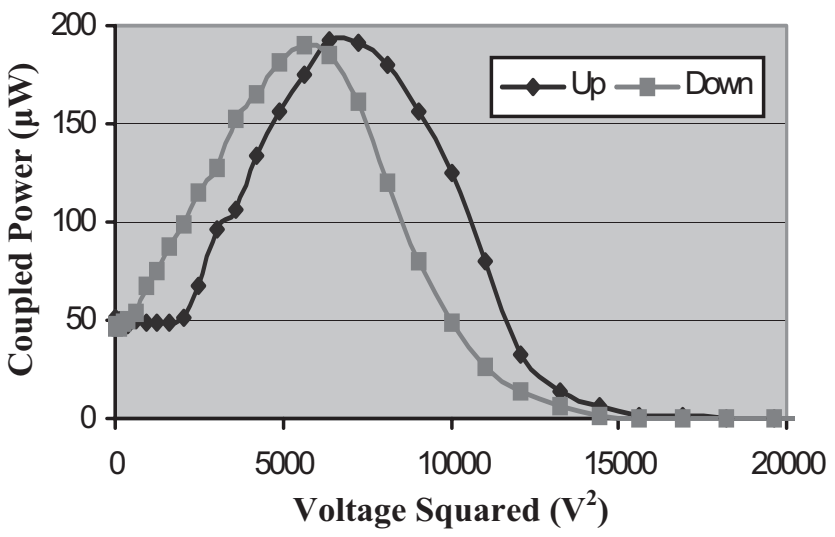

Figure 6: Actuating both sides identically creates a vertical up/down motion that exhibits noticeable hysteresis ( 4 4m). 


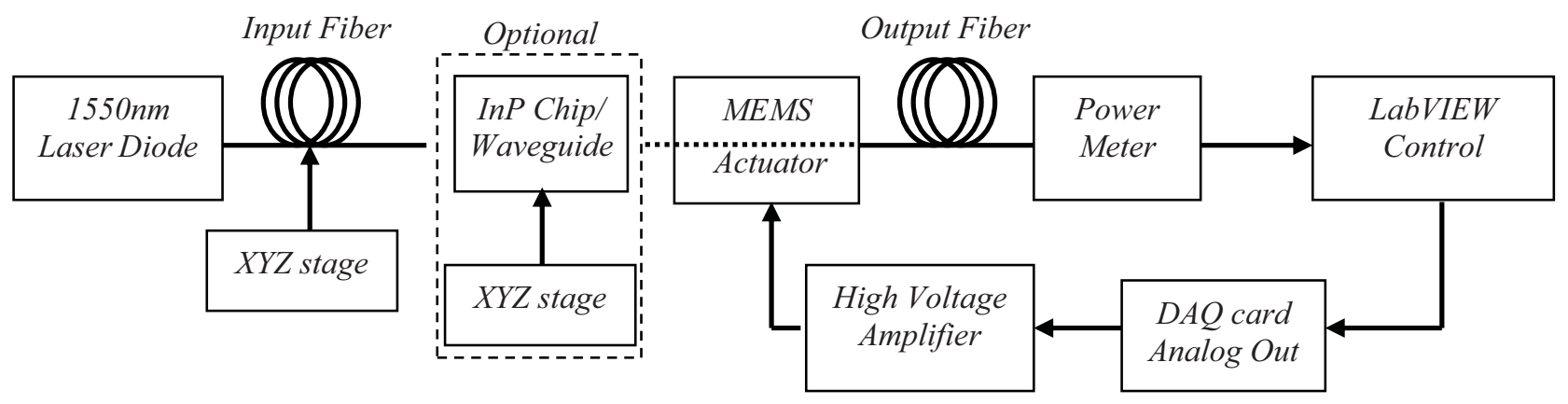

Figure 7: Optical test setup for auto-alignment of MEMS-actuated fiber to cleaved fibers or InP waveguides.

frictional force acts in the opposite direction and delays the fiber's return "Down" to its new state. This hysteresis effect should be reduced by improving the wedge design and/or fabrication. However, fiber alignment using closed-loop control has proven robust with the current structures. Single devices have been actuated $>10^{5}$ times in numerous testing configurations without any observed change in performance.

\section{Automated Alignment}

The optical setup used for automated alignment characterization is shown in Figure 7. A $1550 \mathrm{~nm}$ laser diode is coupled to a fiber located on an electrostrictively-controlled XYZ stage. The MEMS aligner sits on the output stage, with its fiber coupled to an optical power meter to measure the coupled power. Depending on the configuration, an InP waveguide [7] is placed between the two fibers and misaligned by approximately $\sim 20 \mu \mathrm{m}$ with the MEMS aligner. The setup is controlled via LabVIEW.

Alignment algorithms typically contain an initial coarse alignment step to achieve an intermediate threshold power (to avoid noise/secondary peaks), followed by a fine alignment step to optimize the alignment. The simplest coarse alignment routine is that of a raster scan, where the voltage on the $1^{\text {st }}$ actuator is held fixed, while the voltage on the $2^{\text {nd }}$ actuator is swept. The voltage on the $1^{\text {st }}$ actuator is then incremented, and the process repeated. Using a raster scan, Figure 8 shows the time required to achieve coarse alignment as a function of input fiber position. The sloped wedges cause the contour lines to be tilted with respect to the X-Y axes. Note that times $>36 \mathrm{sec}$ indicate failure to achieve threshold, illustrating the diamond-shaped alignment area of the device.

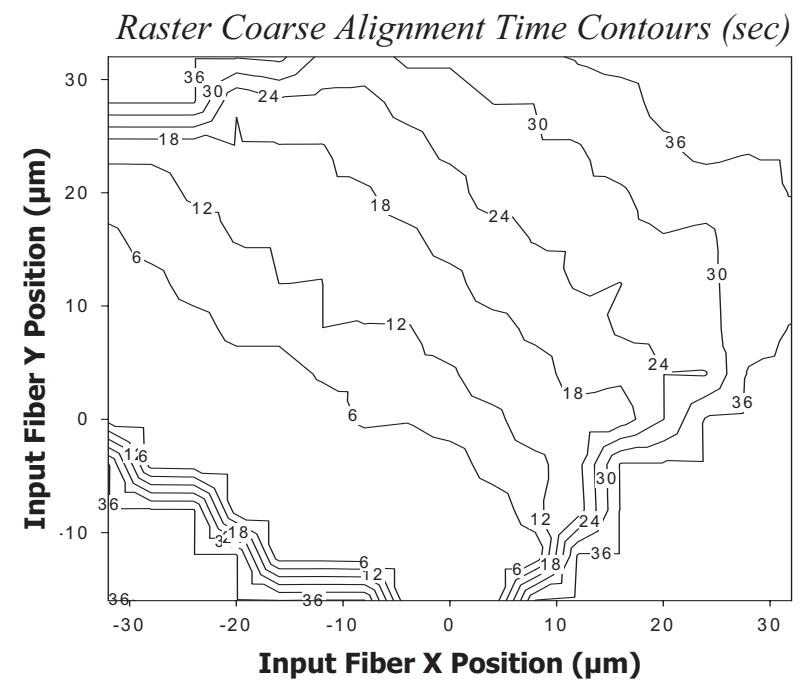

Figure 8: Coarse alignment time contours using a raster algorithm for different locations of the input fiber.
A hill-climbing algorithm [8] was then modified to serve as the fine alignment step. Inserting an InP ribbed waveguide to our setup, Figure 9 shows the total alignment time for different coarse threshold powers and settling times (the pause between discrete fiber movements and power measurements). The final alignment threshold used for this test (92\% peak) corresponds to $\sim 3.5 \mu \mathrm{m}$ misalignment (calibrated using the electrostrictive XYZ stages). We observed that the total alignment time was reduced from 34.2 to 8.5 seconds by decreasing both the settling time (from $1000 \mathrm{~ms}$ to $300 \mathrm{~ms}$ ) and coarse threshold level (from $75 \%$ to $50 \%$ peak). Further decreasing the settling time did not allow sufficient time for the power meter to read accurately, while smaller threshold powers would leave the system susceptible to false peaks. However, in all cases of Figure 9, the coarse algorithm dominates total alignment time and the alignment accuracy $(3.5 \mu \mathrm{m})$ was much larger than desired.

A spiral search algorithm was developed for our actuator to replace the raster scan to decrease coarse alignment time. A $2 \mu \mathrm{m}$ square InP waveguide (tighter optical confinement than ribbed) was also used to decrease the alignment threshold to $1.6 \mu \mathrm{m}$ (in conjunction with increasing the ultimate threshold power to $95 \%$ of the peak). Rather than begin at the rest location of the MEMS aligner like the raster scan, the spiral algorithm begins in the center of the achievable motion, presumably the most likely location for the alignment target (in this case the square InP waveguide). The fiber then proceeds to spiral outward to progressively less-likely positions until the coarse alignment threshold is reached. Figure 10 shows the measured coarse alignment time for different input fiber positions, confirming that the coarse threshold is reached most quickly for targets located near the center. Note that the total

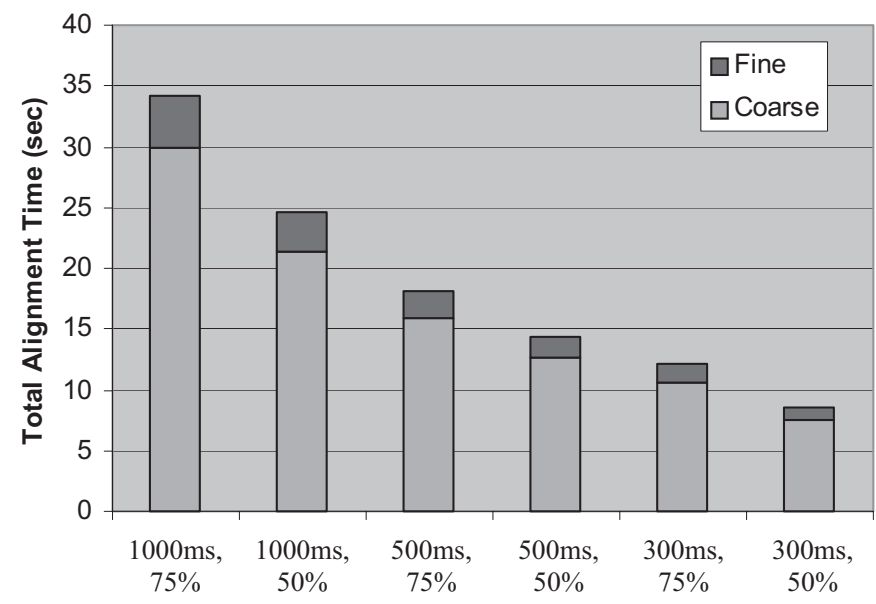

Settling Time, Coarse Threshold Power (\%Peak)

Figure 9: Alignment times to an InP waveguide for different settling times and coarse threshold power (\% peak). 
Spiral Coarse Alignment Time Contours (sec)

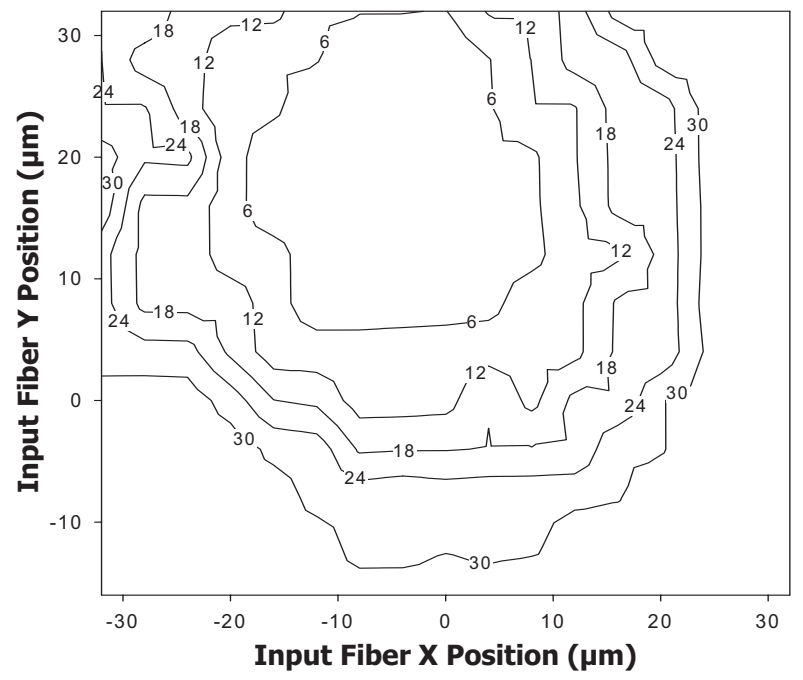

Figure 10: Coarse alignment time contours using a spiral algorithm as a function of input fiber location.

time required to scan the entire alignment area is approximately the same $(>30 \mathrm{sec})$ for both raster and spiral algorithms, with the primary difference between them being the location where they start their search.

Figure 11 compares the total alignment time required to align within $1.6 \mu \mathrm{m}$ of the square InP waveguide using raster or spiral algorithms and different incremental actuator step sizes (Voltage ${ }^{2}$ applied to comb-drive). As expected, using the spiral algorithm dramatically decreased the coarse alignment time for an InP waveguide located towards the center of the range. In addition, we observed that the longer fine alignment time for large $\mathrm{V}^{2}$ increments was caused by often overshooting the optimal position due to the tighter alignment tolerance. Ultimately, an accuracy of $<1.6 \mu \mathrm{m}(>95 \%$ of peak coupled power) was routinely achieved in $5-10$ seconds to InP waveguides initially misaligned by $\sim 20 \mu \mathrm{m}$.

\section{CONCLUSION}

The alignment results reported here compare favorably to active alignment times reported using external actuators [8] while approaching the desired micron-level resolution. The device design, fabrication, and algorithms discussed provide numerous avenues for optimizing active alignment time and accuracy for optoelectronics packaging. Alternative search algorithms could also be adapted to work with this actuator design.

Future work will concentrate on miniaturizing the actuator to enable alignment of fiber arrays in a compact footprint and within optoelectronic packaging modules. Methods for further improving the alignment accuracy of these devices will also be pursued.

\section{ACKNOWLEDGEMENTS}

This research was funded in part by the U.S. Army Research Laboratory under the Collaborative Technology Alliance Power and Energy Program (Cooperative Agreement DAAD-19-01-20010), NASA-Goddard Space Flight Center (Grant APRA040000-0087), and an NSF-CAREER award (Ghodssi). The authors would like to thank the staff of both the Army Research Lab (ARL) and the Laboratory for Physical Sciences (LPS) for access

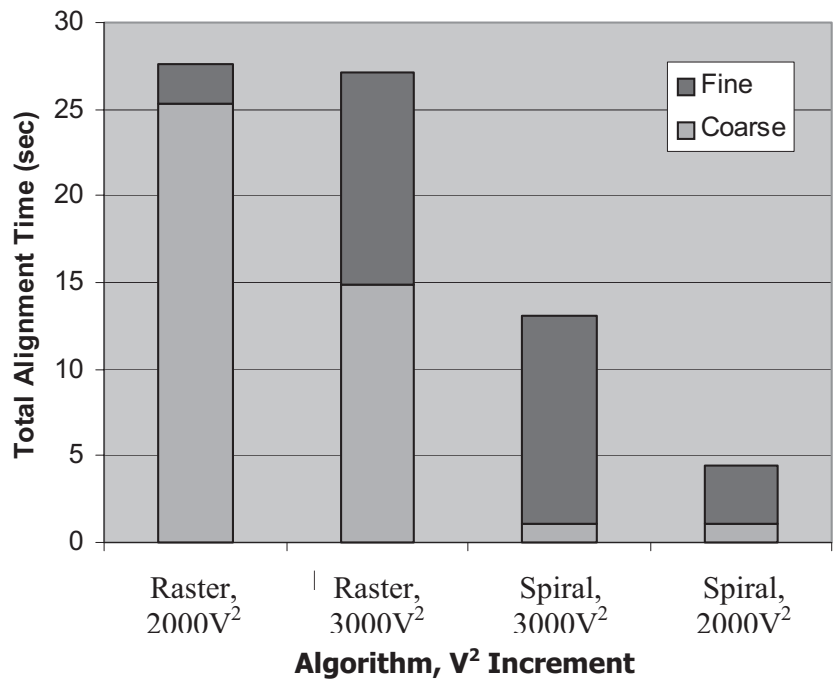

Figure 11: Time to align within $<1.6 \mu m$ (95\% peak power) to an InP waveguide, as a function of coarse search algorithm and Voltage $^{2}$ increment setting.

to their cleanroom facilities, Northrop Grumman for optical mask fabrication, and Jonathan McGee of UMD for instrumentation assistance. Brian Morgan is the recipient of the Achievement Rewards for College Scientists (ARCS) Fellowship (Metropolitan Washington Chapter) for 2004-2005 and 2005-2006.

\section{REFERENCES}

[1] C. A. Armiento, A. J. Negri, M. J. Tabasky, R. A. Boudreau, M. A. Rothman, T. W. Fitzgerald, and P. O. Haugsjaa, "Gigabit transmitter array modules on silicon waferboard," IEEE Transactions on Components, Hybrids, and Manufacturing Technology, vol. 15, pp. 1072, 1992.

[2] W. J. Shakespeare, R. A. Pearson, J. L. Grenestedt, P. Hutapea, and V. Gupta, "MEMS integrated submount alignment for optoelectronics," Journal of Lightwave Technology, vol. 23, pp. 504, 2005.

[3] J. M. Haake, R. L. Wood, and V. R. Dhuler, "In-package active fiber optic micro-aligner," Proceedings of the SPIE, vol. 3276, pp. 207, 1998.

[4] T. Frank, "Development and manufacturing of a twodimensional microactuator for moving of an optical fibre," Proceedings of the SPIE - The International Society for Optical Engineering, vol. 2882, pp. 226, 1996.

[5] B. Morgan, and R. Ghodssi, "On-chip 2-Axis Optical Fiber Actuator using Gray-scale Technology," Proceedings of IEEE MEMS 2006, Istanbul, Turkey, pp. 266-269, 2006.

[6] B. Morgan, C. M. Waits, J. Krizmanic, and R. Ghodssi, "Development of a deep silicon phase Fresnel lens using Gray-scale lithography and deep reactive ion etching," J. of Microelectromechanical Systems, vol. 13, pp. 113, 2004.

[7] D. P. Kelly, M. W. Pruessner, K. Amarnath, M. Datta, S. Kanakaraju, L. C. Calhoun, and R. Ghodssi, "Monolithic suspended optical waveguides for InP MEMS," Photonics Technology Letters, IEEE, vol. 16, pp. 1298, 2004.

[8] J. Guo and R. Heyler, "Fast active alignment in photonics device packaging," 2004 Proceedings. 54th Electronic Components and Technology Conf, vol. Vol.1, pp. 813, 2004. 